\title{
AMCoR
}

Asahikawa Medical College Repository http://amcor.asahikawa-med.ac.jp/

Lancet (2003. Dec) 362(9399):1918-1920.

Human Taeniasis and cysticercosis in Asia

Ito, Akira ; Nakao, Minoru ; Wandra, Toni 


\section{Human taeniasis and cysticercosis in Asia}

Akira Ito, Minoru Nakao, Toni Wandra

Context Human taeniases caused by the pork tapeworm, Taenia solium and by the beef tapeworm, Taenia saginata are meat-borne parasitic diseases, and require pork and beef contaminated with metacestodes, the larval stage of these parasites, respectively. Taeniasis of $T$. solium has serious medical or public health problems, since it simultaneously causes cysticercosis, especially neurocysticercosis (NCC), threatening human life through accidental ingestion of eggs released from taeniasis patients. Approximately $70 \%$ of human population who has the custom to eat pork is risky for taeniasis/cysticercosis of $T$. solium. In contrast, taeniasis of $T$. saginata appears to be more widely distributed and common than T. solium. Over several decades it has been generally noticed that adult taeniid tapeworms expelled from local people in Asia appeared to be $T$. saginata (so called Asian Taenia), although they ate pork. It is now named Taenia asiatica and has been found from Taiwan, Korea, China, Vietnam and Indonesia, at least. (152 words) 
Starting point Recent work on taeniasis in Asia indicates a third taeniid species, Taenia asiatica ( $\mathrm{AL}$ Willingham and others. SE Asian J Trop Med Pub Health 2003; 34 (Suppl 1) : 35-50) which is still unfamiliar even for the majority of parasitologists. Phylogenetic analysis of Taenia spp. and historical, ecological and biogeographic analyses by EP Hoberg and others (Proc Roy Soc London (B) 2001; 268: 781-787) has indicated that $T$. asiatica is an independent species and the sister species of $T$. saginata and strongly suggested that (i) a molecular clock (conservative) estimate indicates divergence of these sister species, $T$. asiatica and $T$. saginata, at a minimum > 160, 000 years ago, and (ii) T. solium is only distantly related and emerged in Africa several million years ago as a parasite unique to Homo erectus. M Nakao and others (Parasitology 2002; 124: 657-662) provided the first "clock" evidence for the timing of the speciation event of two (Asian and American/African) genotypes of $T$. solium worldwide. Molecular techniques available for phylogeny, evolution, diagnosis by several groups including the authors' group and vaccination by MW Lightowlers and other (Int J Parasitol 2003; 33: 1207-17) against taeniasis and cysticercosis of human taeniid infections have greatly advanced over the past decade. It, therefore, is timely to review these all. (210 words) 
Where next Molecular and immunological techniques including vaccine and development of experimental animal models for differentiation of taeniid species in humans have greatly advanced over the past decade. What is clinical importance of infections by these taeniids in humans including $T$. asiatica? Where are they distributed in Asia? What is the potential for cysticercosis attributable to $T$. asiatica in humans? And what is the present situation for cysticercosis linked to $T$. solium in Asia? (72 words)

(Total words for peg: 434 words)

Department of Parasitology, Asahikawa Medical College, Midorigaoka-Higashi 2-1-1-1, Asahikawa 078-8510, Japan (A Ito, Ph.D.; M Nakao, Ph.D.)

Directorate General Communicable Disease Control and Environmental Health, Ministry of Health, Jakarta, Indonesia (T Wandra, M.D.)

Correspondence to: Prof. A Ito (e-mail: akiraito@asahikawamed.ac.jp) 
The life history of Asian Taenia was experimentally demonstrated by Fan and his colleagues in Taiwan. ${ }^{1}$ In contrast to $T$. saginata, pigs are required intermediate hosts, and infective metacestodes, or cysticerci of small dimensions (e.g., $2 \mathrm{~mm}$ in diameter) develop in the liver and survive for a relatively short period of 2-3 months. The metacestodes in pigs have only rudimentary or vestigial hooks, but strobilate adults lack rostellar armature and in this regard are similar to $T$. saginata. It is clear that Asian Taenia is biologically unique. However, if it was found to be related to $T$. solium rather than T. saginata, it might have indicated the potential for human cysticercosis. ${ }^{2}$

Eom and $\mathrm{Rim}^{3}$ described and differentiated Asian Taenia based on comparative morphology and designated a new species, Taenia asiatica. The most recent phylogenetic studies based on mitochondrial DNA (mtDNA) or comparative morphology have shown the validity and independence of $T$. asiatica as the sister species of $T$. saginata, both are distantly related to $T$. solium. ${ }^{4-7}$ Major characteristics of three species of Taenia that develop as adults in human definitive hosts are summarized in Table 1.8

Asian Taenia is Taenia asiatica, the sister species of Taenia saginata 
Additional evidence supporting this concept is no hybrid of $T$. asiatica and $T$. saginata found in sympatric zones in China. ${ }^{9,10}$ Thus, if these two sister species are discrete biological entities, with separate histories, as indicated by phylogeny and an array of diagnostic criteria, ${ }^{3-8}$ and they are now sympatric, ${ }^{9,10}$ then $T$. asiatica can not justifiably be reduced as a subspecies of $T$. saginata. There is just one minor comment on the lack of definitive cross breeding experiments. ${ }^{11,12}$ Species definition aside, the multiple lines of evidence for $T$. asiatica support it an independent, sister species of $T$. saginata.

\section{Where is $T$. asiatica distributed in Asia?}

This parasite has been known to be present in Taiwan, Korea, China (several provinces), Indonesia (North Sumatra) and Vietnam at least where local people have the custom to eat uncooked pork with viscera after killing pigs at home. ${ }^{1,}{ }^{13}$

It has been reported that $T$. saginata, $T$. asiatica and $T$. solium coexist in Guangxi Autonomous Region and in Yunnan Province, China and in Vietnam. ${ }^{9,10,14}$

\section{Is the pig suitable intermediate host of $T$. asiatica?} Based on the stunted cysticerci of $T$. asiatica in pigs, there is some doubt if pigs are the really suitable intermediate hosts 
or if this parasite has well adapted to use pigs as its intermediate hosts.

When non-obese diabetic severe combined immunodeficient (NOD/Shi-scid) mice were used for preparation of cysticerci of these three taeniid species, oncosphere embryos of $T$. asiatica and $T$. saginata developed into cysticerci of approximately 10 $\mathrm{mm}$ in diameter with similar morphology and differed from those of T. solium. ${ }^{15}$

\section{Does cysticercosis of $T$. asiatica exist in humans?}

Based solely on this association demonstrated in both molecular and morphological studies, it is predicted that cysticercosis in humans attributable to $T$. asiatica does not occur, because it is a phenomenon unknown in its sister-species, $T$. saginata. ${ }^{2,5-7}$

\section{Taenia solium worldwide}

Recent topics on Evolution and two genotypes of $T$. solium The most serious taeniid species for humans is $T$. solium, since it induces neurocysticercosis in humans. Hoberg and others ${ }^{6}$ launched a hypothesis that (i) $T$. solium emerged in Africa several million years ago (MYA) as a parasite of Homo erectus and came out from Africa, (ii) T. solium is only distantly related with $T$. saginata, and has its own independent 
evolutionary trajectory and historical ecology. Recently, Nakao and others ${ }^{16-18}$ indicated that (i) T. solium in the world can be divided into Asian and African/American genotypes which diverged approximately 1 MYA in western Paleactic (Europe) and Asia, (ii) the tapeworm was maintained and diverged in early human colonies through a 'human-human' cycle (cannibalism) and a 'human-wild suids' cycle (hunting), and (iii)European (western Paleactic) genotype was introduced into Africa, southern par of the Sahara desert, and America through European exploration and colonization since 1500s. These two research groups stress that $T$. solium is originally human parasite through cannibalism. Although there is no critical evidence of pathological differences between Asian and African/American genotypes, it is crucial to identify two genotypes of $T$. solium, since it travels with human carriers. ${ }^{11,18,19}$

\section{T. solium taeniasis/cysticercosis in Asia}

The highly endemic areas in Asia locate everywhere the local people consume pork: China, India, Vietnam, Lao PD, Cambodia, Nepal, Myanmar, Philippines, Indonesia where local pigs roam around with free access to human feces. From Japan and South Korea, it has been eradicated. ${ }^{20,21}$ Situations in India and Indonesia are of particular interest. The majority of people in India are vegetarians but NCC with solitary cyst is very 
common. ${ }^{20,22}$ By contrast, the majority of people in Indonesia are Muslim but NCC is spreading in several islands where the majority is Hindu or Christian. ${ }^{20,23,24}$

\section{T. solium taeniasis/cysticercosis in Papua, Indonesia}

The present situation in Papua (= former Irian Jaya), Indonesia appears to be the worst in the world. There are several brief reviews on the history of introduction of NCC into Papua. ${ }^{22,24}$ It has been revealed that approximately $46 \%$ of local people $\geqq$ 18 years old examined in Jayawijaya District in 1996-1997 were expected to be cysticercosis of $T$. solium, either symptomatic or asymptomatic. ${ }^{24,26}$ Approximately $>8 \%$ and $32 \%$ of local people living in the local capital city, Wamena, Jayawijaya examined in 2001 harbored adult tapeworms of $T$. solium and expected to be suffering from cysticercosis, respectively. ${ }^{27}$ The genotype of $T$. solium in Papua has been confirmed to be the Asian type using expelled proglottids, eggs and subcutaneous cysts resected. 24,26,27 Local people live with pigs and dogs together and these pigs and dogs function as scavengers and therefore highly infected with cysticerci of $T$. solium. ${ }^{28,29}$ As eating dog meat is still not rare in Asia, especially in Korea, China, Vietnam and some parts of Indonesia including North Sumatra. Some ethnics living in Java, Sulawesi, Papua have custom to eat dog meat. Therefore, there might be some contribution for 
maintenance of the life history of $T$. solium through the dog-human cycle as well as the well-known the pig-human cycle. ${ }^{29}$

It is urgent for us to check the situation of $T$. solium taeniasis/cysticercosis in other islands in Indonesia and the neighboring countries including East Timor and Papua New Guinea. ${ }^{24}$

Present situation of taeniasis in Sumatra and taeniasis/cysticercosis in Bali

In North Sumatra, the ethnic groups living in Samosir Island in the Lake Toba are Christians and used to eat uncooked minced pork with viscera and blood ${ }^{13}$ similar in Bali where local Hindi people still eat uncooked minced pork with fresh blood and recently have started eating of uncooked minced beef too (Wandra et al. unpublished). ${ }^{23}$ There is no crucial evidence of taeniasis/cysticercosis of $T$. solium in Samosir Island and taeniasis of $T$. asiatica, which has well been recognized as endemic disease there $\mathrm{e}^{13}$, is now not so common in 2003 (Wandra et al. unpublished). Similarly, taeniasis/cysticercosis of $T$. solium which was common in Bali one decade ago $^{30}$ is becoming rare. In contrast, a rapid increase in the number of taeniasis of $T$. saginata with prevalence rate of more than $20 \%$ of local people in several villages has most recently become evident (Wandra et al, unpublished). There is no critical evidence of 
T. asiatica in humans in Bali so far. It is interesting to understand the situation in these two areas in Indonesia. It is strongly suggested that taeniasis/cysticercosis of $T$. solium in Bali and taeniasis of $T$. asiatica in North Sumatra have become not common through changes in pig husbandry to keep pigs indoor without direct contact with human feces, improvement of sanitation, and sustainable education to boil pork before eat (Wandra et al. unpublished).

\section{Importance of identification of human Taenia}

It is actually most important to know who is infected with $T$. solium due to the issue of neurocysticercosis (NCC) in humans. This drives the necessity to accurately and rapidly identify any species of Taenia in a human definitive host; and to accurately identify any cysticerci resulting from recovery of metacestodes from infected humans.

It is crucial to identify and differentiate $T$. asiatica from $T$. saginata for epidemiological or public health concerns. The life cycle of $T$. asiatica appears to be completed in rather remote areas where pigs can roam around with free access to human feces in many Southeast Asian countries as similar as that of T. solium in Asia, Latin America and Africa. ${ }^{31}$

The most accurate approaches for identification of species are based on total evidence whether molecular and immunological 
diagnostics are combined with comparative morphology. ${ }^{7,33}$ Multiplex PCR to identify $T$. asiatica, $T$. saginata, and Asian and Africa/America genotypes of $T$. solium is now available. ${ }^{10,32}$ Serology for cysticercosis in humans, pigs and dogs are available and serology for taeniasis in humans as well as copro-antigen test and copro-DNA test for taeniasis are available. ${ }^{33}$ The human cases of taeniasis of $T$. saginata in the world, especially at least in Asia should be re-evaluated based on mtDNA and epidemiological study for differentiation of $T$. asiatica. Much of this material may not be accessible by current molecular techniques due to methods of preservation, or the possibility that specimens no longer exist. Thus, it would be necessary to engage in new and broad-based surveys across this region.

\section{Prevention and control of human Taeniasis}

Clinical manifestation of taeniasis of $T$. asiatica appears to be similar to that of $T$. saginata but differs from that of $T$. solium. Almost every day, singled proglottids of either $T$. asiatica or $T$. saginata actively and spontaneously come out from our anus. It is the most common symptom that tapeworm carriers themselves easily recognize by themselves. Therefore for diagnosing of outpatients and for epidemiological survey, it is important to show expelled proglottids for demonstration. 
Treatment with a single dose of praziquantel or nicrosamide is sufficient to expel adult worms of $T$. saginata or $T$. asiatica or even $T$. solium. ${ }^{21}$

There are two strategies for control of taeniasis other than improvement of meat inspection. The first is for foreign visitors and local people in endemic areas not to eat such local food with uncooked or undercooked meat with viscera. The second is to keep pigs indoor with no contact with human feces which has greatly been proven for prevention of cysticercosis of $T$. solium in developed countries ${ }^{31}$ and in Bali. Cut-off the life cycle of $T$. solium may depend on sustainable public health education ( $i$ ) not to eat uncooked or undercooked but well cooked meat or viscera with improvement of meat inspections, and (ii) to block the direct contact of domestic animals with human feces through improvement of sanitation and the style of husbandry of animals.

Alternative strategy for control of cysticercosis is through vaccination and chemotherapy of domestic animals. ${ }^{33,34}$ Recent work on vaccination of pigs and cattle against $T$. solium and T. saginata, respectively, is highly promising. ${ }^{34}$ It is expected to be informative if the gene homology of vaccine candidate proteins between $T$. saginata and $T$. asiatica is very high, it may work against $T$. asiatica too. Another strategy for control of $T$. solium is chemotherapy of pigs by single dose 
of oxfendazole. ${ }^{33,35}$ These techniques for domestic animals and treatment of humans infected with Taenia should be carried out simultaneously and under sustainable financial support with sustainable education of the local people living with these parasites. ${ }^{33}$

Conclusion Current evidence would support recognition of $T$. asiatica as a valid species that is distinct from $T$. saginata. It has been found from Taiwan, Korea, Indonesia, Vietnam and China so far where local people or the ethnic groups eat uncooked home-made pork with viscera. A sister-species relationship for $T$. asiatica and $T$. saginata and the predictive nature of phylogenetic information suggest that cysticercosis in humans attributable to the former species is unlikely. (1940 words)

\section{Addendum}

For such international collaborations through technical transfer, the Ministry of Education, Japan (MEXT) has decided for the senior author to establish the network for control of taeniasis/cysticercosis and echinococcosis in Asia from 2003 for three years.

Supported by grants from the Ministry of Education, Japan 
(060344089, 07044243, 09044279, 10557029, 11694259) and the Japan Society for Promotion of Science $(12557024,14256001)$ to AI. We all sincerely thank for the reviewers who have improved this review with highly useful suggestions and comments.

\section{References}

1. Fan PC. Taiwan Taenia and taeniasis. Parasitol Today 1988; 4:86-88.

2. Ito A. Cysticercosis in Asian-Pacific regions. Parasitol Today 1992; 8: 181-82.

3.Eom K, Rim HJ. Morphological descriptions of Taenia asiatica sp. Korean J Parasitol 1993; 31: 1-6.

4.Queiroz A, Alkire NL. The phylogenic placement of Taenia cestodes that parasitize humans. J Parasitol 1998; 84: 379-83.

5. Hoberg EP, Jones A, Rausch RL, et al. A phylogenetic hypothesis for species of the genus Taenia (Eucestoda: Taeniidae). J Parasitol 2000; 86: 89-98.

6. Hoberg EP, Alkire NL, De Queiroz A, Jones A. Out of Africa: origin of the Taenia tapeworms in humans. Proc Roy Soc London B 2001; 268: 781-87.

7.Hoberg EP. Taenia tapeworms: their biology, evolution and socioeconomic significance. Microbes Infect 2002; 4: 
$859-66$.

8.Pawlowski ZS. Chapter 1. Taenia solium: Basic biology and transmission. In Singh G, Prabhakar S (Eds), Taenia solium Cysticercosis. CABI Press, 0xson, pp.1-13, 2002.

9.Eom KS, Jeon HK, Kong Y, et al. Identification of Taenia asiatica in China: molecular, morphological, and epidemiological analysis of a Luzhai isolate. J Parasitol $2002 ; 88: 758-64$.

10. Yamasaki H, Allan JC, Sato MO, et al. DNA differential diagnosis of taeniasis/cysticercosis by multiplex PCR. J Clin Microbiol 2003; 41: in press.

11. Ito A, Yamasaki H, Nakao M, et al. Multiple genotypes of Taenia solium-ramifications for diagnosis, treatment and control. Acta Trop 2003; 87: 95-101.

12. Nakao M, Sako, Y, Ito A. Isolation of polymorphic microsatellite loci from the tapeworm Echinococcus multilocularis. Inf Genet Evol 2003; 3: 159-63.

13. Kosin E, Depary A, Djohansjah A. Taeniasis di pulau Samosir. Maj. Kedok. Univ. Sumatra Utara, Medan 1972; 3: 5-11.

14.Willingham AL, De NV, Doanh NQ, et al. Current status of cysticercosis in Vietnam. SE Asian J Trop Med Pub Health $2003 ; 34$ (Suppl 1): 35-50.

15. Ito A, Nakaya K, Sako $Y$, et al. NOD-scid mouse as an experimental animal model for cysticercosis. SE Asian J 
Trop Med Pub Health 2001; 32 (Suppl 2): 85-89.

16. Nakao M, Sako Y, Yokoyama N, et al. Mitochondrial genetic code in cestodes. Mol Biochem Parasitol 2000; 111: 415-24.

17. Nakao M, Yokoyama N, Sako Y, et al. The complete mitochondrial DNA sequence of the cestode Echinococcus multilocularis (Cyclophyllidea: Taeniidae). Mitochondrion 2002; 1: 497-509.

18. Nakao M, Okamoto M, Sako Y, et al. A phylogenetic hypothesis for the distribution of two genotypes of the pig tapeworm Taenia solium worldwide. Parasitology 2002; 124: 657-62. 19.European Commission, Health and Consumer Protection Directorate General. Opinion of the scientific committee on veterinary measures relating to public health on "The control of taeniosis/cysticercosis in man and animals" (adopted on September 28-29, 2000). http://Www.europa.eu. int/comm/food/fs/sc/scv/.

20.Singh G, Prabhakar S, Ito A, et al. Chapter 12. Taenia solium taeniasis and cysticercosis in Asia. In Singh G, Prabhakar S (Eds), Taenia solium Cysticercosis. CABI Press, 0xson, pp. $111-27,2002$.

21. Ito A, Urbani C, Qiu JM, et al. Control of echinococcosis and cysticercosis: a public health challenge to international cooperation in China. Acta Trop 2003; 86: $3-17$. 
22. Rajshekhar V, Chandy MJ. Solitary cysticercus granuloma. Orient Longman, Hyderabad, 169pp, 2000.

23. Simanjuntak GM, Margono SS, okamoto M, Ito A. Taeniasis/cysticercosis in Indonesia as an emerging disease. Parasitol Today 1997; 13:321-23.

24.Wandra T, Ito A, Yamasaki $H$, et al. Taenia solium cysticercosis in Irian Jaya, Indonesia. Emerg Infect Dis $2003 ; 9: 884-5$.

25.Gujdusek DC. Introduction of Taenia solium into west New Guinea with a note on an epidemic of burns from cysticercosis epilepsy in the Ekari people of the Wissel Lakes area. PNG Med J 1978; 21: 329-42.

26. Wandra T, Subahar R, Simanjuntak GM, et al. Resurgence of cases of epileptic seizures and burns associated with cysticercosis in Assologaima, Jayawijaya, Irian Jaya, Indonesia, 1991-95. Trans $R$ Soc Trop Med Hyg 2000; $94: 46-50$.

27.Margono SS, Ito A, Sato Mo, et al. Taenia solium taeniasis/cysticercosis in Papua, Indonesia in 2001: detection of human worm carriers. J Helminthol 2003; 77: $39-42$.

28. Subahar R, Hamid A, Purba $W$, et al. Taenia solium infection in Irian Jaya (West Papua), Indonesia: a pilot serological survey of human and porcine cysticercosis in Jayawijaya 
District. Trans $R$ Soc Trop Med Hyg 2001; 95: 388-90.

29. Ito A, Putra MI, Subahar $R$, et al. Dogs as alternative intermediate hosts of Taenia solium in Papua (Irian Jaya), Indonesia confirmed by highly specific ELISA and immunoblot using native and recombinant antigens and mitochondrial DNA analysis. J Helminthol 2002; 76: 311-14.

30. Sutisna IP, Fraser A, Kapti IL, et al. Community prevalence study of taeniasis and cysticercosis in Bali, Indonesia. Trop Med Internat Health 1999; 4: 288-94.

31. Schantz PM, Wilkins PP, Tsang VCW. Immigrants, imaging and immunoblots: the emergence of neurocysticercosis as a significant public health problem. In Scheld WM, Craig WA, Hughes JM (Eds), Emerging Infections 2. ASM Press, Washington DC, pp. 213-42, 1998.

32. Yamasaki $H$, Nakao $M$, Sako $Y$, et al. DNA differential diagnosis of human taeniid cestodes by base excision sequence scanning thymine-base reader analysis with mitochondrial genes. J Clin Microbiol 2002; 40: 3818-21.

33. Ito A, Craig PS. Immunodiagnosis and molecular approaches for the detection of taeniid cestode infections. Trends Parasitol 2003; 19: 377-81.

34. Lightowlers MW, Gauci CG, Chow C, et al. Molecular and genetic characterization of the host-protective oncosphere antigens of taeniid cestode parasites. Int $\mathrm{J}$ Parasitol 
2003; 33: 1207-17.

35. Gonzalez AE, Garcia HH, Gilman RH, et al. Control of Taenia solium. Acta Trop 2003; 87: 103-09. 
Table 1. Several major characteristics of three species of human Taenia (modified from ${ }^{8,15}$ )
Characteristics
T. solium
T. asiatica
T. saginata

\section{Cysticercus}

Intermediate hosts (IH)

pigs, humans ${ }^{\mathrm{a}}$, dogs, wild boar

muscle, brain, skin, eye, tongue

rostellum with hooklets

Number of uterine branches

in a gravid proglottids

Expulsion from human
$7-12$

mainly in groups, passively viscera mainly liver

$2 \times 2^{b}$

rostellum with rudimentary hooks

rostellum with no hooks

$16-21$

pigs, cattle, goat, monkey, wild boar

attle, reindeer ${ }^{c}$

muscle, viscera, brain ${ }^{c}$

$7-10 \times 4-6$

no rostellum, no hook

no rostellum, no hook

18-32

single, actively single, actively

a: neurocysticercosis is the most serious.

b: the size and morphology of cysticercus of $T$. asiatica becomes to be similar to that of $T$. saginata in NOD/Shi-scid mice. ${ }^{15}$

${ }^{c}$ : cysticerci are found from the brain of the reindeer. 